\title{
Gene therapy for cystic fibrosis: which postman, which box?
}

\author{
Peter G Middleton, Eric W F W Alton
}

\begin{abstract}
Since 1989 when the gene responsible for cystic fibrosis was cloned and designated the cystic fibrosis transmembrane conductance regulator (CFTR) gene, considerable progress has been made in the development of gene therapy for this disease. Clinical trials have already been performed using cationic liposome and adenoviral based gene transfer systems, measuring the safety and efficacy of this new form of treatment, with variable results to date. These two approaches and the current progress in airway gene delivery are discussed.

(Thorax 1998;53:197-199)
\end{abstract}

Keywords: cystic fibrosis; gene therapy; adenovirus; liposome

The clinical syndrome of cystic fibrosis comprising recurrent sinobronchial infections, pancreatic fibrosis, bowel dysfunction, and increased sweat electrolytes is well known to most respiratory physicians. The CFTR gene codes for a protein called CFTR which functions both as a $\mathrm{Cl}^{-}$channel and a regulator of other channels. With the demonstration that delivery of normal copies of the CFTR gene to airway epithelial cells in vitro could reverse the defect in $\mathrm{Cl}^{-}$secretion, methods to transfer the CFTR gene to the airways of human subjects in vivo were developed over the next 3-4 years. Four studies were published initially, three using adenovirus constructs ${ }^{1-3}$ and one liposome-DNA complexes. ${ }^{4}$ All used topical delivery directly to the airways (nasal and/or bronchial), with the aim of maximising delivery of the therapeutic gene to the airway epithelium. However, all of these studies have their limitations and further modifications are likely to be necessary before gene therapy will become standard treatment for patients with cystic fibrosis.

\section{Which postman?}

Gene delivery to humans can be achieved using either ex vivo or in vivo strategies. In the former, progenitor cells such as the bone marrow are harvested and maintained in tissue culture under sterile conditions. Gene transfer is performed into these cells in vitro and the transformed cells are then returned to the individual. However, in view of the complex anatomy of the airways and the sparsity of progenitor cells, ex vivo transfer is generally regarded as unsuitable for respiratory diseases. Gene delivery to the airway epithelium will most probably require the use of in vivo gene transfer.

Gene therapy in vivo can be performed using viral or non-viral systems. In both cases the plasmid DNA comprises three essential elements: the complementary DNA (cDNA) of interest, and both 5' (upstream) and 3' (downstream) flanking regions. The 5' region, commonly called the promoter sequence, regulates the transcription of the introduced gene, whilst the 3' flanking region influences the RNA splicing, polyadenylation, post-translational processing, and enhancing elements. Although these elements are synthesised from virus components, they do not code for proteins and have not been associated with adverse reactions.

\section{LIPOSOME MEDIATED GENE TRANSFER}

Synthetic cationic liposomes have specifically been designed for use as gene transfer agents. They appear most effective at delivering DNA to cells when manufactured as a combination of cationic (positively charged) and neutral lipids. The lipid mixture spontaneously forms vesicles which then bind with the negatively charged DNA. This interaction was initially attributed to simple charge effects, but recent work using electron microscopy has demonstrated that the lipid molecules cover the DNA, causing it to collapse into tight coils. ${ }^{5}$

DNA-liposome complexes have been used widely to investigate the function of the CFTR gene in vitro, and have been delivered directly to the airways of cystic fibrosis mice by nebulisation. ${ }^{6}$ No adverse reactions were seen, suggesting that this form of therapy may be safe in humans. Based on this work, we then studied the safety ${ }^{7}$ and efficacy ${ }^{4}$ of liposomes to transfer the CFTR gene to the nasal epithelium of patients with cystic fibrosis. Following gene delivery there were no adverse reactions and the $\mathrm{Cl}^{-}$secretory response increased significantly, correcting approximately $20 \%$ of the difference between cystic fibrosis and noncystic fibrosis values. Several further studies 
have now confirmed these findings in the nose using a variety of cationic liposomes, and we have recently undertaken a study examining the safety and efficacy of gene delivery to the lower airways using this technique.

\section{ADENOVIRAL VECTORS}

These gene transfer systems exploit the natural viral mechanisms to express genes within human cells. Adenoviruses are large doublestranded DNA viruses which are thought to enter cells through binding of the "fibre" protein to cell surface receptors. The virion is then engulfed into clathrin coated pits and internalised into endosomes. By rupturing the endosome it escapes into the cytoplasm and is transported to the nucleus. Although adenoviral vectors have been shown in vitro to transfer gene constructs to cells effectively, recent evidence has questioned the safety of these vectors for gene therapy in vivo. Studies in baboons have demonstrated gene expression but also a transient decrease in total white blood cell count and dose dependent alveolar inflammation, perivascular lymphocytic infiltrates, and chest radiograph abnormalities. This inflammatory reaction appeared to develop progressively over the 2-3 weeks following gene administration and was thought to be due to expression of viral antigens. Similarly, rhesus monkeys have developed systemic antibodies following delivery of adenoviral constructs to the nasal cavity. To address this difficulty, new vectors have been designed with further viral deletions which appear to lessen viral gene expression, diminishing the inflammatory reaction and also prolonging expression of the transgene. ${ }^{8}$

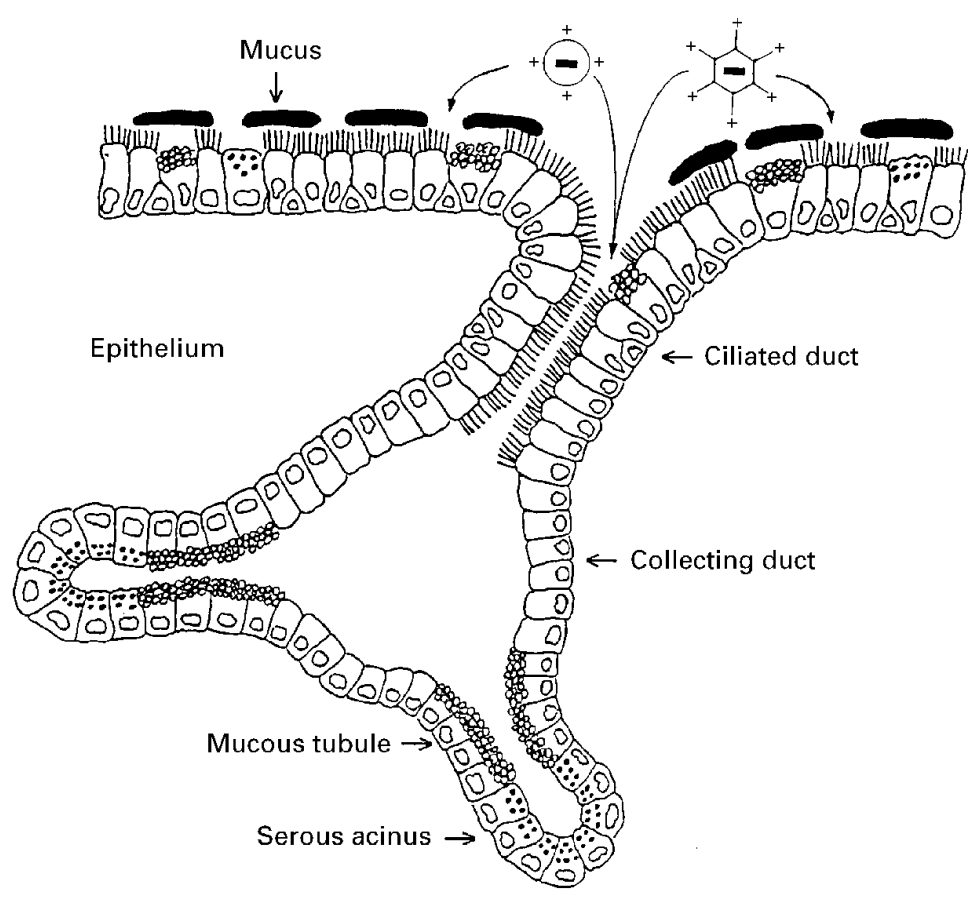

Figure 1 Cross section of the respiratory epithelium showing a submucosal gland opening onto the surface epithelium. The cationic liposome and adenoviral-based gene transfer agents are shown in the airway lumen and will need to cross the airway mucous layer to reach the airway epithelial cells. The important question remains: will topical delivery to the airway lumen generate sufficient gene expression to cure cystic fibrosis?
Clinical trials with adenoviruses have given variable results in patients with cystic fibrosis. The first trial was an open study in three subjects which demonstrated that local delivery to the nasal epithelium was associated with expression of CFTR mRNA. Increased $\mathrm{Cl}^{-}$ secretion and decreased $\mathrm{Na}^{+}$absorption were also seen, ${ }^{1}$ but the physiological techniques used to measure these responses in vivo have been questioned. ${ }^{39}$ A second study, delivering the adenovirus construct to both the nasal and bronchial epithelium of four patients with cystic fibrosis, demonstrated CFTR mRNA and protein expression in some subjects. ${ }^{2}$ Finally, a recent double blind study in 12 patients with cystic fibrosis demonstrated no correction of the defective $\mathrm{Cl}^{-}$secretion. ${ }^{3}$ Thus it would appear that gene transfer, both via adenovirus and liposome-plasmid constructs, will require further refinement before it can be used clinically to treat cystic fibrosis.

\section{Therapeutic potential}

The importance of these preliminary studies is the demonstration that gene delivery to the airway epithelium of patients with cystic fibrosis is possible. The therapeutic potential is the correction of affected cells and thus the prevention of the disease processes. However, there are a number of assumptions in the current use of the $\mathrm{Cl}^{-}$secretory response as an end point for the physiological correction. The relationship between this and clinical benefit is unclear, highlighting the need for answers to several important clinical questions.

\section{Which box?}

Which cell(s) need to express the inserted gene? The predominant expression of CFTR in the airways is in the submucosal glands, yet all the current protocols have used delivery techniques aimed at maximising gene expression in the surface epithelium. If correction of these glands is required to prevent disease progression, then it is unlikely that topical delivery of the gene construct will succeed (fig 1). Other approaches, such as intravenous gene delivery, combined with techniques to target the submucosal glands may be superior. ${ }^{10}$

Is correction of the $\mathrm{Cl}^{-}$secretory response in every cell required for clinical benefit, or would partial correction of some cells be sufficient? Two studies are relevant to this question. The first addressed the proportions of cells which need to express CFTR. Corrected and uncorrected cystic fibrosis cells were mixed together in various proportions, grown into monolayers, and assayed for $\mathrm{Cl}^{-}$transport. As the $\mathrm{Cl}^{-}$transport across monolayers of $6-10 \%$ corrected cells was similar to that of $100 \%$ corrected cells, it was concluded that genetic "correction" of $6-10 \%$ of surface epithelial cells would be sufficient to demonstrate the beneficial effects of gene transfer. ${ }^{11}$ The second study addressed the minimum level of CFTR expression required. By interbreeding cystic fibrosis mouse models which have different levels of CFTR it was demonstrated that approximately $5 \%$ of normal CFTR mRNA (in all the cells) 
can markedly increase $\mathrm{Cl}^{-}$transport and prevent the death of these animals from intestinal disease. ${ }^{12}$

Finally, how do the physiological abnormalities found in cystic fibrosis epithelia relate to the disease processes? The strong link between cystic fibrosis and defective $\mathrm{Cl}^{-}$secretion is well known, but in view of the abnormalities in $\mathrm{Na}^{+}$ absorption, bacterial adherence and local production of defensins, the primary $\mathrm{Cl}^{-}$defect may not be the only important factor.

\section{Conclusion}

In summary, in vivo gene therapy to human airways has been reported for both liposomal and adenoviral gene transfer. Both systems have demonstrated proof of principle, and progress is steady and encouraging. The development of new gene delivery systems is proceeding rapidly, including the use of adenoassociated virus, receptor conjugates, and combined viral-liposome approaches. However, further investigations into the pathophysiology of cystic fibrosis are required to clarify the appropriate target cells for gene therapy. Ironically, many of the questions about the pathogenesis of cystic fibrosis may well be answered by the development of gene therapy for this disease.
1 Zabner J, Couture LA, Gregory RJ, et al. AdenovirusZabner J, Couture LA, Gregory RJ, et al. Adenovirus-
mediated gene transfer transiently corrects the chloride mediated gene transfer transiently corrects the chloride
transport defect in nasal epithelia of patients with cystic transport defect in nasal epithe

2 Crystal RG, McElvaney NG, Rosenfeld MA, et al. Administration of an adenovirus containing the human CFTR cDNA to the respiratory tract of individuals with cystic fibrosis. Nature Genet 1994;8:42-51

3 Knowles MR, Hohneker KW, Zhou Z, et al. A controlled study of adenoviral-vector-mediated gene transfer in the nasal epithelium of patients with cystic fibrosis. $N$ Engl f Med 1995;333:823-31.

4 Caplen NJ, Alton EWFW, Middleton PG, et al. Liposomemediated CFTR gene transfer to the nasal epithelium of patients with cystic fibrosis. Nature Med 1995;1:39-46.

5 Gershon H, Ghirlando R, Guttman SB, et al. Mode of formation and structural features of DNA-cationic liposome complexes used for transfection. Biochemistry 1993; 32:7143-51.

6 Alton EWFW, Middleton PG, Caplen NJ, et al. Noninvasive liposome-mediated gene delivery can correct the ion transport defect in cystic fibrosis mutant mice. Nature Genet 1993;5:135-42.

7 Middleton PG, Caplen NJ, Gao X, et al. Nasal application of the cationic liposome DC-Chol:DOPE does not alter ion transport, lung function or bacterial growth. Eur Respir $\mathcal{F}$ 1994;7:442-5.

8 Yang Y, Nunes FA, Berencsi K, et al. Inactivation of E2a in recombinant adenoviruses improves the prospect for gene therapy in cystic fibrosis. Nature Genet 1994;7:362-9.

9 Middleton PG, Geddes DM, Alton EWFW. Protocols for in vivo measurement of the ion transport defects in cystic fibrosis nasal epithelium. Eur Respir f 1994;7:2050-6.

10 Ferkol T, Perales JC, Eckman E, et al. Gene transfer into the airway epithelium of animals by targeting the polymeric immunoglobulin receptor. $\mathcal{F}$ Clin Invest 1995;95:493-502.

immunoglobulin receptor. F Clin Invest 1995;95:493-502.
11 Johnson LG, Olsen JC, Sarkadi B, et al. Efficiency of gene Johnson LG, Olsen JC, Sarkadi B, et al. Efficiency of gene
transfer for restoration of normal airway epithelial function transfer for restoration of normal airway epithe

12 Dorin JR, Farley R, Webb S, et al. A demonstration using mouse models that successful gene therapy for cystic fibrosis requires only partial gene correction. Gene Therapy 1996;3:797-801. 